\title{
MAXIMAL AND MINIMAL TOPOLOGIES $\left({ }^{1}\right)$
}

BY

\author{
DOUGLAS E. CAMERON
}

\begin{abstract}
A topological space $(X, T)$ with property $\mathrm{R}$ is maximal $\mathrm{R}$ (minimal $\mathrm{R}$ ) if $T$ is a maximal (minimal) element in the set $\mathrm{R}(X)$ of all topologies on the set $X$ having property $\mathbf{R}$ with the partial ordering of set inclusions. The properties of maximal topologies for compactness, countable compactness, sequential compactness, Bolzano-Weierstrass compactness, and Lindelöf are investigated and the relations between these spaces are investigated. The question of whether any space having one of these properties has a strictly stronger maximal topology is investigated. Some interesting product theorems are discussed. The properties of minimal topologies and their relationships are discussed for the quasi- $P$, Hausdorff quasi- $P$, and $P$ topologies.
\end{abstract}

I. Background and introduction. For a given topological property $\mathrm{R}$ and a set $X$, we let $\mathrm{R}(X)$ denote the set of all topologies on $X$ which have property $\mathrm{R}$ and observe that $\mathrm{R}(X)$ is partially ordered by set inclusion. A topological space $(X, T)$ is maximal $\mathrm{R}$ ( $\mathrm{R}$-maximal) provided that $T$ is a maximal element in $\mathrm{R}(X)$. A topological space $(X, T)$ is minimal $\mathrm{R}$ ( $\mathrm{R}$-minimal) if $T$ is a minimal element in $\mathrm{R}(X)$. A topology $T^{\prime}$ on the set $X$ is finer than a topology $T$ if $T^{\prime} \supseteq T$; the topology $T$ is said to be coarser than the topology $T^{\prime}$.

The concept of minimal topologies was first introduced in 1939 by A. S. Parhomenko [19] when he showed that compact Hausdorff spaces are minimal Hausdorff. Four years later E. Hewitt [12] proved that compact Hausdorff spaces are maximal compact as well as being minimal Hausdorff. In 1947 R. Vaidyanathaswamy [33] asked if there existed noncompact minimal Hausdorff spaces or non-Hausdorff maximal compact spaces. The same year A. Ramanathan [22], [23] showed that such minimal Hausdorff spaces existed and characterized all minimal Hausdorff spaces. In 1948 Hing Tong [30] constructed an example of a maximal compact space which was not Hausdorff and thus answered the other part of Vaidyanathaswamy's question.

In 1948 A. Ramanathan [24] proved that a topological space is maximal compact

Received by the editors June 29, 1970 and, in revised form, November 18, 1970.

AMS 1970 subject classifications. Primary 54A10, 54B05, 54B10, 54C20, 54D20, 54D25, 54D30, 54D35; Secondary 54B05, 54D60.

Key words and phrases. Maximal topologies, minimal topologies, compactness, countable compactness, sequential compactness, Bolzano-Weierstrass compactness, Lindelöf, quasi- $P$ spaces, Hausdorff quasi- $P$-spaces, $P$-spaces, strongly $\mathrm{R}$ topologies.

( ${ }^{1}$ Most of this research is part of the author's dissertation at Virginia Polytechnic Institute, Blacksburg, Virginia, under the direction of C. E. Aull. The author wishes to thank the referee for his comments and suggestions which greatly enhanced the structure of the paper.

Copyright (C) 1971, American Mathematical Society 
if and only if its compact subsets are precisely the closed sets. Not much work was done concerning maximal properties until 1963 when N. Smythe and C. A. Wilkins [26] constructed an example of a maximal compact space without isolated points which is strictly weaker than a minimal Hausdorff topology. In 1965 N. Levine [18] proved that products of maximal compact spaces are not necessarily maximal compact when he showed that $(X \times X, T \times T)$ is maximal compact if and only if $(X, T)$ is Hausdorff. He also showed that the one point compactification of the rationals with the usual topology is maximal compact but is not Hausdorff.

In his book Topological structures, W. J. Thron proved that a first countable Hausdorff countably compact space is maximal countably compact and minimal first countable Hausdorff. A strengthening of this result was obtained by C. E. Aull [2] when he showed that a countably compact $E_{1}$ space is maximal countably compact and minimal $E_{1}$ (a topological space is $E_{1}$ if each point is the countable intersection of closed neighborhoods). J. R. Porter [20] has recently shown that minimal $E_{1}$ spaces are precisely the minimal Hausdorff first countable spaces discussed by R. M. Stephenson, Jr. [27]. In 1968 J. Pelham Thomas [28] published a paper discussing maximal connected topologies.

The study of minimal properties has been much more intense than the study of maximal properties. The author suggests that for a background on the development and results of minimal property research, the reader refer to $A$ survey of minimal topological structures by M. P. Berri, J. R. Porter, and R. M. Stephenson, Jr. [6].

One of the questions investigated in the study of minimal properties is when does a space have a coarser topology which is minimal $R$ ? If a space has this property, it is said to be Katetov R. For example, it has been shown that the rational numbers with the usual topology is not Katetov Hausdorff [7]. The corresponding question to be asked for maximal properties is when does a topological space have a finer topology which is maximal $R$ ? If a topological space has this property we shall say that the space is strongly $\mathrm{R}$. Among the results obtained in relation to this question, we shall show that not all Lindelöf spaces are strongly Lindelöf and that not all Bolzano-Weierstrass compact spaces are strongly BolzanoWeierstrass compact.

The notation and definitions used shall be standard with the convention accepted that no separation axioms will be attached to any other property unless specifically stated. Wherever a nonstandard definition or notation is to be used, it shall be introduced in the text. The most frequently used notations are the following: the closure of a set $A$ with respect to the topology $T$ is $\operatorname{cl}_{T} A$; the interior of a set $A$ with respect to the topology $T$ is int $_{T} A$; the relative topology of the set $A$ with respect to the topology $T$ is $T / A$; the complement of $A$ in $X$ is $X-A$; and the open neighborhood system of $x$ with respect to the topology $T$ is $V_{T}(x)$. If $(X, T)$ is a topological space and $A \notin T$, then $T(A)=\left\{U_{1} \cup\left(U_{2} \cap A\right): U_{1}, U_{2} \in T\right\}$ is the topology on $X$ generated by the subbase $\{A\} \cup T ; T(A)$ is called the simple extension of $T$ by $A$. 
II. General results. J. E. Joseph [14] proved that in a compact space $(X, T)$ every compact subset is closed if and only if every continuous bijection from a compact space $(Y, V)$ to $(X, T)$ is a homeomorphism. We shall generalize this to obtain the following theorem.

THEOREM 2.1. A topological space is maximal $\mathrm{R}$ if and only if every continuous bijection from a space $(Y, V)$ with property $\mathrm{R}$ to $(X, T)$ is a homeomorphism.

Proof. If $(X, T)$ is maximal and $f:(Y, V) \rightarrow(X, T)$ is a continuous bijection, then for $T^{\prime}=\{f(G): G \in V\}, f:(Y, V) \rightarrow\left(X, T^{\prime}\right)$ is a homeomorphism, and $\left(X, T^{\prime}\right)$ has property $\mathrm{R}$. Since $T^{\prime} \supseteq T$ and $(X, T)$ is maximal $\mathrm{R}, T^{\prime}=T$.

If $(X, T)$ is not maximal $\mathrm{R}$, then there is $T^{\prime} \supset T$ such that $\left(X, T^{\prime}\right)$ has property $\mathrm{R}$. The identity map $I:\left(X, T^{\prime}\right) \rightarrow(X, T)$ is a continuous bijection which is not a homeomorphism.

A topological property $\mathrm{R}$ is contractive if $(X, T)$ has property $\mathrm{R}$ and if $T^{\prime} \subseteq T$, then $\left(X, T^{\prime}\right)$ has property $\mathrm{R}$. A property $\mathrm{R}$ is closed hereditary if all closed subsets of a space with property $\mathrm{R}$ also have property $\mathrm{R}$.

A topological property $\mathrm{R}$ has condition

$(\alpha)$ if $(X, T)$ has property $\mathrm{R}$ and $A \subseteq X$ has property $\mathrm{R}$, then $(X, T(X-A))$ has property $\mathbf{R}$;

$(\beta)$ if a single point space has property $\mathrm{R}$.

The covering axioms of compactness, countable compactness and Lindelöf as well as Bolzano-Weierstrass compactness, sequential compactness and connectedness are all contractive, closed hereditary and satisfy conditions $(\alpha)$ and $(\beta)$.

THEOREM 2.2. A topological property $\mathrm{R}$ is contractive if and only if it preserved under continuous bijections.

THEOREM 2.3. If $\mathrm{R}$ is a contractive topological property, then $(X, T)$ is maximal $\mathrm{R}$ if and only if for $A \notin T,(X, T(A))$ does not have property $\mathrm{R}$.

THEOREM 2.4. Let property $\mathrm{R}$ be contractive, closed hereditary and have property $(\alpha)$. A topological space $(X, T)$ is maximal $\mathrm{R}$ if and only if the subsets with property $\mathrm{R}$ are precisely the closed sets.

Proof. Since the space is closed hereditary, all closed sets have property $\mathrm{R}$. If $A \subseteq X$ has property $\mathrm{R}$ but is not closed, then $T(X-A) \supset T$ has property $\mathrm{R}$ and so $(X, T)$ is not maximal. If $(X, T)$ is not maximal then there is $T^{\prime} \supset T$ such that ( $X, T^{\prime}$ ) has property $\mathrm{R}$ and there is $A \notin T$ such that $T \subset T(A) \subseteq T^{\prime}$ and $T(A)$ has property $\mathrm{R}$; therefore $X-A$ has property $\mathrm{R}$ and is not closed in $(X, T)$.

COROLlARY 2.1. If property $\mathrm{R}$ is contractive, closed hereditary and satisfies $(\alpha)$ and $(\beta)$, then the maximal $\mathrm{R}$ spaces are $T_{1}$.

THEOREM 2.5. If property $\mathrm{R}$ is contractive, closed hereditary and satisfies condition $(\alpha)$, then in maximal spaces the $\mathrm{R}$ subsets are maximal $\mathrm{R}$ in their relative topologies. 
Proof. If $(X, T)$ is maximal $\mathrm{R}, A \subseteq X$, and $(X, T / A)$ has property $\mathrm{R}$, then $A$ is closed in $(X, T)$. If $B \subseteq A$ and $B$ has property $\mathrm{R}$, then $B$ is closed in $T$ and thus $B$ is closed in $T / A$.

$\mathrm{N}$. Levine [18] proved the following three theorems for compactness.

THEOREM 2.6. Let property $\mathrm{R}$ be contractive, closed hereditary and satisfy conditions $(\alpha)$ and $(\beta)$. If $\left(\prod_{B} X_{\beta}, \prod_{B} T_{\beta}\right)$ is maximal $\mathrm{R}$, then $\left(X_{\beta}, T_{\beta}\right)$ is maximal for each $\beta \in B$.

Proof. Since the continuous image of a space with property $\mathrm{R}$ also has property $\mathrm{R},\left(X_{\beta}, T_{\beta}\right)$ has property $\mathrm{R}$ for each $\beta \in B$. Let $A$ be an $\mathrm{R}$ subset of $\left(X_{\alpha}, T_{\alpha}\right)$ for some $\alpha \in B$. Choose $x_{\beta} \in X_{\beta}$ for $\beta \neq \alpha$, and let $A_{\beta}=\left\{x_{\beta}\right\}$ for $\beta \neq \alpha$ and $A_{\alpha}=A$. Then $\prod_{B} A_{\beta}$ is an $\mathrm{R}$ subset of $\left(\prod_{B} X_{\beta}, \prod_{B} T_{\beta}\right)$ and so it is closed. Then since $\operatorname{cl}_{\prod_{B} T_{\beta}}\left(\prod A_{\beta}\right)$ $=\prod\left(\mathrm{cl}_{T_{\beta}} A\right)=\prod_{B} A_{\beta}, A$ is closed in $\left(X_{\alpha}, T_{\alpha}\right)$.

THEOREM 2.7. Let property $\mathrm{R}$ be contractive, closed hereditary and satisfy condition $(\alpha)$. If $(X, T)$ is $\mathrm{R}$, then $(X \times X, T \times T)$ is maximal $\mathrm{R}$ only if $(X, T)$ is Hausdorff.

Proof. If $(X \times X, T \times T)$ is maximal $\mathrm{R},\{(x, x): x \in X\}$ is closed since it is homeomorphic to $(X, T)$ and so $(X, T)$ is Hausdorff.

The case of general products is not completely resolved and shall be discussed in other sections.

THEOREM 2.8. Let property $\mathrm{R}$ be contractive, closed hereditary and satisfy condition ( $\alpha$ ). If $(X, T)$ is maximal $\mathrm{R}, X=\bigcup\left\{A_{\beta}: \beta \in B\right\}$, and $\left(A_{\beta}, T / A_{\beta}\right)$ has property $\mathrm{R}$ for each $\beta \in B$, then $A_{\beta}$ is $T$ closed and $\left(A_{\beta}, T / A_{\beta}\right)$ is maximal $\mathrm{R}$ for each $\beta \in B$.

The converse of the preceding theorem does not hold in general since if a property $\mathrm{R}$ satisfies condition $(\beta)$, then every space is the union of its maximal $\mathrm{R}$ subspaces. However, the following result is a special case of the theorem in which the converse is valid.

COROLlaRY 2.2. Let $\mathrm{R}$ be contractive, closed hereditary and satisfy condition $(\alpha)$, and satisfy the condition that a space which is the finite union of subspaces with property $\mathrm{R}$ also has property $\mathrm{R}$. If $B$ is a finite set, $X=\bigcup\left\{A_{\beta}: \beta \in B\right\}$, and $\left(A_{\beta}, T / A_{\beta}\right)$ has property $\mathrm{R}$ for each $\beta \in B$, then $(X, T)$ is maximal $\mathrm{R}$ if and only if $A_{\beta}$ is $T$ closed and $\left(A_{\beta}, T / A_{\beta}\right)$ is maximal $\mathrm{R}$ for each $\beta \in B$.

Let $X$ be a set and $T$ and $T^{\prime}$ two topologies on $X$. Then $T \vee T^{\prime}$ is the topology on $X$ which has as a subbase the topologies $T$ and $T^{\prime}$; the topology $T \vee T^{\prime}$ is the least upper bound topology with respect to $T$ and $T^{\prime}$.

If $\mathrm{R}$ is a contractive property and $(X, T)$ is strongly $\mathrm{R}$, then $(X, T)$ has property $\mathrm{R}$.

THEOREM 2.9. Let property $\mathrm{R}$ be contractive, closed hereditary and satisfy conditions $(\alpha)$ and $(\beta)$. A space $(X, T)$ with property $\mathrm{R}$ is strongly $\mathrm{R}$ only if $(X, T \vee F)$ is strongly $\mathbf{R}$ where $F$ is the topology of finite complements. 
Proof. Corollary 2.1 states that maximal $\mathrm{R}$ spaces are $T_{1}$ and so if $\left(X, T^{\prime}\right)$ is maximal $\mathrm{R}, T \subseteq T^{\prime}$, then $T^{\prime} \supseteq F$ and therefore $T^{\prime} \supseteq T \vee F$.

THEOREM 2.10. Let property $\mathrm{R}$ be contractive, closed hereditary and satisfy condition $(\alpha)$. Strongly $\mathrm{R}$ is closed hereditary.

THEOREM 2.11. Let property $\mathrm{R}$ be contractive, closed hereditary and satisfy condition $(\alpha)$. If the product of $T_{1}$ spaces is strongly $\mathrm{R}$, then each coordinate space is strongly $\mathbf{R}$.

The condition that the spaces be $T_{1}$ is important not only in the method of proof, but also since $\left(\prod_{A} T_{\alpha}\right) \vee F \neq \prod_{A}\left(T_{\alpha} \vee F\right)$.

THEOREM 2.12. Let property $\mathrm{R}$ be contractive, closed hereditary and satisfy condition $(\alpha)$. If some infinite product of $T_{1}$ spaces of more than one point is strongly $\mathbf{R}$, then the Cantor set is strongly $\mathbf{R}$.

Proof. Such a product contains a closed subspace homeomorphic to a countably infinite product of discrete spaces with two points and hence to the Cantor set. An application of Theorem 2.10 completes the proof.

\section{Maximal compactness.}

EXAmple 3.1. Let $X$ be an infinite set, $x_{0} \in X$. $\{x\}$ is open for all $x \neq x_{0}$ and $x_{0} \in G$ is open if $X-G$ is finite. This is maximal compact and is a modification of an example in Gillman and Jerison [11] (see Example 7.2).

The following was proved by E. Hewitt [12].

THEOREM 3.1. A compact Hausdorff space is maximal compact and minimal Hausdorff.

We observe that all locally compact Hausdorff spaces and all completely regular Hausdorff spaces are embeddable in maximal compact Hausdorff spaces-their one point and Stone-Čech compactifications respectively.

Corollary 2.1 states that all maximal compact spaces are $T_{1}$. The example by Hing Tong [30] shows that all maximal compact spaces are not Hausdorff; also the one point compactification of the rationals with the usual topology is maximal compact but not Hausdorff since the rationals are not locally compact [18]. Hausdorff quotients of compact spaces are maximal compact, but the following example shows that $T_{1}$ quotients of maximal compact spaces are not necessarily maximal compact.

EXAMPLE 3.2. Let $\left(Y_{i}, T_{i}\right)$ be the one point compactification of $N$ with the discrete topology and $y_{i}$ the added point for $i=1,2$. Let $(X, T)$ be the free union of $\left(Y_{1}, T_{1}\right)$ and $\left(Y_{2}, T_{2}\right)$. Then $(X, T)$ is maximal compact. Let $X^{*}=\left\{y_{1}, y_{2}\right\} \cup N$ and $T^{*}$ the topology on $X^{*}$ having as a base of open sets $\{n\}$ for $n \in N,\left\{y_{1}\right\} \cup(N-A)$ and $\left\{y_{2}\right\} \cup(N-B)$ where $A$ and $B$ are arbitrary finite subsets of $N$. This space is $T_{1}$ and is not maximal compact since $\left\{y_{1}\right\} \cup N$ is compact but is not closed. 
IV. Maximal countable compactness. Example 3.2 is a maximal countably compact space as well as being maximal compact.

We have characterized the maximal countably compact spaces as those countably compact spaces in which the countably compact subsets are precisely the closed sets; the following theorem characterizes these spaces in terms of sequences.

THEOREM 4.1. A countably compact space $(X, T)$ is maximal countably compact if and only if, for every $G \notin T$, there is a sequence $S \subseteq X-G$ with no adherent points in $X-G$.

Proof. A space is not countably compact if and only if it contains a sequence with no adherent points.

THEOREM 4.2. For countable spaces, maximal countable compactness is equivalent to maximal compactness.

A topological space is an $E_{0}$ space if every point is a $G_{\delta}$. A topological space is an $E_{1}$ space if every point is the intersection of a countable number of closed neighborhoods.

It is easily seen that $E_{1}$ spaces are Hausdorff and that first countable Hausdorff spaces are $E_{1}$. C. E. Aull [2] has given an example to show that $E_{0}$ spaces do not have to be Hausdorff.

The following was proved by C. E. Aull [2].

THEOREM 4.3. Every countably compact $E_{1}$ space is maximal countably compact and minimal $E_{1}$.

An alternate way of stating this theorem is that every Hausdorff first countable countably compact space is maximal countably compact and minimal Hausdorff first countable since J. R. Porter [20] has shown that minimal $E_{1}$ spaces are precisely the same as the minimal Hausdorff first countable spaces discussed by R. M. Stephenson, Jr. [27].

COROLlARY 4.1. In an $E_{1}$ space, all the countably compact subsets are closed.

EXAMPLE 4.1. The ordinals less than the first uncountable ordinal with the usual topology is countably compact and $E_{1}$ and is therefore maximal countably compact and minimal $E_{1}$ and is not compact.

EXAMPLE 4.2. The ordinals less than or equal to the first uncountable ordinal with the usual topology is a Hausdorff compact space and is therefore maximal compact. It is a countably compact space but is not maximal countably compact since the space of Example 4.1 is a countably compact subspace which is not closed. The simple extension of this topology by $\{\Omega\}, \Omega$ being the first uncountable ordinal, is maximal countably compact and is not compact.

Examples 4.1 and 4.2 show that the maximal countably compact and the maximal compact spaces are not the same; however a maximal countably compact space which is compact is maximal compact. 
EXAMPLE 4.3. If $(X, T)$ is a Hausdorff completely regular space, then the StoneČech compactification $\beta X$ is maximal compact but is not necessarily maximal countably compact; for example, $\beta N$ where $N$ is the natural numbers with the usual topology. The author does not know conditions under which $\beta X$ is maximal countably compact.

EXAMPLE 4.4. This is an example of a maximal countably compact space which is not Hausdorff and has no isolated points. The example was originally constructed by N. Smythe and C. A. Wilkins [26] as an example of a maximal compact space which is strictly weaker than a minimal Hausdorff space.

Let $X=\{A, B\} \cup R$ where $R$ is the real numbers. The topology $T$ on $X$ is determined as follows: $x \in R$ has the usual neighborhood base; the neighborhood base for $A$ consists of all sets of the form

$$
U_{n}=\{A\} \cup\{(2 r-1,2 r):|r| \geqq n, r \text { an integer }\}
$$

for some positive integer $n$; the neighborhood base for $B$ consists of all sets of the form

$$
V_{\left(n, d_{r}\right)}=\{B\} \cup\left\{\left(2 r-d_{r}, 2 r+1+d_{r}\right):|r| \geqq n, r \text { is an integer }\right\}
$$

for some positive integer $n$ and $d_{r}>0, d_{r}$ varies with each $r$ and with each $n$.

Example 4.5. Let $X=\{A, B\} \cup R$ with the neighborhood bases for $A$ and the points in $R$ the same as in Example 4.4. The neighborhood base for the point $B$ consists of all sets of the form

$$
V_{n}=\{B\} \cup\{(2 r, 2 r+1):|r| \geqq n, r \text { an integer }\}
$$

for some positive integer $n$.

N. Smythe and C. A. Wilkins [26] proved that this space is minimal Hausdorff and since it is also first countable, it is minimal first countable Hausdorff or minimal $E_{1}$. Thus this space is a minimal $E_{1}$ space which is strictly stronger than the maximal countably compact space of Example 4.4.

We may observe that the space of Example 4.4, while not $E_{1}$, is $E_{0}$ and first countable.

EXAMPLE 4.6. The uncountable space of Example 3.1 is maximal countably compact but not first countable nor $E_{0}$.

THEOREM 4.4. The countable product of countably compact $E_{1}$ spaces is maximal countably compact.

Proof. J. R. Porter [20] has shown that countably compact $E_{1}$ spaces are first countable and so are sequentially compact. Since the countable product of $E_{1}$ spaces is $E_{1}$ and the countable product of sequentially compact spaces is sequentially compact, the theorem follows.

The preceding result does not extend to arbitrary products since $\beta N$ is embeddable as a closed subset of products of $[0,1]$ with the usual topology and $\beta N$ is not maximal countably compact. 
We observed in $\$ 3$ that the one point compactification of a locally compact Hausdorff space is maximal compact; however, it is not necessarily maximal countably compact (Example 4.2).

Let $(X, T)$ be a noncountably compact space; $X^{*}=X \cup\left\{x_{0}\right\}, x_{0} \notin X ; T^{*}$ $=T \cup\left\{G \subseteq X^{*}: x_{0} \in G\right.$ and $X-G$ is a closed countably compact subset in $\left.(X, T)\right\}$. $\left(X^{*}, T^{*}\right)$ is the one point countable compactification of $(X, T)$. We want to answer the question: When is the one point countable compactification of a noncountably compact space maximal countably compact? We shall obtain at least a partial answer to this question by considering local countable compactness.

There are three possible definitions for local countable compactness in keeping with the literature:

(i) Every point has a countably compact neighborhood.

(ii) Every point has a neighborhood whose closure is countably compact.

(iii) Every neighborhood of a point contains a neighborhood whose closure is countably compact.

A space satisfying (iii) also satisfies (ii); a space satisfying (ii) also satisfies (i); and an $E_{1}$ space satisfying (i) also satisfies (iii).

THEOREM 4.5. The one point countable compactification of a locally countably compact $E_{1}$ space is maximal countably compact but is not necessarily $E_{0}$.

Proof. First we observe that the local countable compactness means that $\left(X^{*}, T^{*}\right)$ is Hausdorff. If $A$ is a countably compact subset of $X^{*}$ and $x_{0} \notin A$, then $A$ is countably compact as a subset of $X$ and is therefore closed and thus closed in $X^{*}$. If $x_{0} \in A$, let $x \notin A$ and $\left\{U_{n}: n \in N\right\}$ be open sets such that $\bigcap\left\{\operatorname{cl}_{T} U_{n}: n \in N\right\}$ $=\{x\}$. Then there exists $U_{n}^{\prime} \subseteq U_{n}$ such that $\mathrm{cl}_{T} U_{n}^{\prime}$ is countably compact for each $n \in N$. Thus $\left\{X^{*}-\operatorname{cl}_{T} U_{n}^{\prime}: n \in N\right\}$ is a $T^{*}$ open cover of $A$ and has a finite subcover; so, there is an open set $U$ containing $x$ such that $U \cap A=\varnothing$. Therefore $A$ is closed and so $\left(X^{*}, T^{*}\right)$ is maximal countably compact. Example 3.1 with $X$ uncountable is the one point countable compactification of $X-\left\{x_{0}\right\}$ and it is not $E_{0}$.

The preceding theorem gives a sufficient but not necessary condition for the one point countable compactification to be maximal countably compact, since the one point countable compactification of the rationals with the usual topology is maximal countably compact but is not Hausdorff since the rationals are not locally countably compact. This is easily verified in the same manner as $\mathbf{N}$. Levine [18] showed that the one point compactification of the rationals is maximal compact since in countable spaces compact and countably compact are equivalent.

Finally in our discussion of maximal countably compact spaces we state the following theorem proved by C. E. Aull [4] concerning limits of convergent sequences.

THEOREM 4.6. In a maximal countably compact space every convergent sequence has a unique limit. 
V. Maximal Bolzano-Weierstrass compactness. We showed in Theorem 2.4 that a Bolzano-Weierstrass compact space is maximal Bolzano-Weierstrass compact if and only if the Bolzano-Weierstrass compact sets are precisely the closed sets and in Corollary 2.1 that these spaces are $T_{1}$. Therefore the characterization of maximal Bolzano-Weierstrass compact spaces is completed with the following theorem, since in a $T_{1}$ space, Bolzano-Weierstrass compactness is equivalent to countable compactness.

THEOREM 5.1. A space is maximal Bolzano-Weierstrass compact if and only if it is maximal countably compact.

COROLlaRY 5.1. A space is strongly Bolzano-Weierstrass compact if and only if it is strongly countably compact.

COROllaRY 5.2. A strongly Bolzano-Weierstrass space is countably compact.

All Bolzano-Weierstrass compact spaces are not strongly Bolzano-Weierstrass compact since there are Bolzano-Weierstrass compact spaces which are not countably compact.

VI. Maximal sequential compactness. We proved in Theorem 2.4 that a sequentially compact space is maximal if and only if the sequentially compact subsets are precisely the closed sets. C. E. Aull [3] was the first to prove this fact, and in [4] he showed that, in a space in which the sequentially compact sets are closed, convergent sequences have unique limits; and in a sequentially compact space in which convergent sequences have unique limits and the sequentially compact sets are closed, the countably compact sets are closed. Therefore we obtain the following results.

THEOREM 6.1. In a maximal sequentially compact space convergent sequences have unique limits.

We observe that the condition of Theorem 6.1 is not a sufficient condition for maximal sequential compactness since the ordinals less than or equal to the first uncountable ordinal are sequentially compact but are not maximal sequentially compact.

THEOREM 6.2. A sequentially compact space is maximal sequentially compact if and only if it is maximal countably compact.

The author does not know whether there are maximal countably compact spaces which are not sequentially compact.

THEOREM 6.3. In an $E_{1}$ space maximal countable compactness is equivalent to maximal sequential compactness.

CoRollary 6.1. An $E_{1}$ space is strongly countably compact if it is strongly sequentially compact. 
In his dissertation in 1906, M. Fréchet [9] defined the limit of a sequence in an abstract space and also defined an $\mathscr{L}$-space:

An abstract set of elements is an $\mathscr{L}$-space if to certain sequences $\left(p_{n}\right)$ of elements of the set there corresponds an element $p=\lim _{n \rightarrow \infty} p_{n}$ called the limit of the sequence such that the following three conditions are satisfied:

(i) limits are unique;

(ii) if $\lim _{n \rightarrow \infty} p_{n}=p$ and $k_{1}<k_{2}<\cdots$, then $\lim _{n \rightarrow \infty} p_{k_{n}}=p$;

(iii) if for every $n, p_{n}=p$, then $\lim _{n \rightarrow \infty} p_{n}=p$.

In $1923 \mathrm{P}$. Alexandrov and P. Urysohn [1] defined $\mathscr{L}^{*}$-spaces as $\mathscr{L}$-spaces which satisfy the additional condition:

(iv) if $\left(p_{n}\right)$ does not converge to $p$, it contains a subsequence $\left(p_{k_{i}}\right)$ none of whose subsequences converge to $p$.

The closure $X^{-}$of a set $X$ in an $\mathscr{L}^{*}$-space is defined as follows: $p \in X^{-}$if and only if $p$ is the limit of a sequence of elements of $X$. It is easily seen that the resulting closure operator satisfies the following conditions:

(i') $(X \cup Y)^{-}=X^{-} \cup Y^{-}$;

(ii') $X \subseteq X^{-}$;

(iii') $\phi^{-}=\phi$; and

(iv') $\{p\}^{-}=\{p\}$.

This operator would be a Kuratowski closure operator if in addition to (i')-(iii') it satisfied the additional condition:

(v') $X^{--} \subseteq X^{-}$.

Thus if an $\mathscr{L}^{*}$-space satisfies $\left(\mathrm{v}^{\prime}\right)$ it is a $T_{1}$ topological space.

C. Kuratowski [15] defines a countably compact $\mathscr{L}^{*}$-space to be an $\mathscr{L}^{*}$-space in which every infinite sequence of points $\left(p_{n}\right)$ contains a convergent subsequence. It has been shown that in an $\mathscr{L}^{*}$-topological space this definition agrees with the usual definition of sequential compactness. It is easily shown that in a countably compact $\mathscr{L}^{*}$-topological space, every closed set is countably (or sequentially) compact and every countably (or sequentially) compact set is maximal sequentially compact. We shall now consider the converse of the above statement; that is: Is every maximal sequentially compact space an $\mathscr{L}^{*}$-space? We shall see by Example 6.1 that the answer to this question is in the negative.

P. Urysohn [31] showed in 1926 that in order for a $T_{1}$ topological space to be an $\mathscr{L}^{*}$-space it was necessary and sufficient that the following two conditions be satisfied:

(i*) every convergent sequence has a unique limit;

(ii*) if $x \in \mathrm{cl}_{T} A$ then there is a sequence $\left(x_{n}\right)$ of points in $A$ such that $\lim _{n \rightarrow \infty} x_{n}$ $=x$.

We have already shown that in a maximal sequentially compact space every convergent sequence has a unique limit point. The following example shows that the second condition of Urysohn does not necessarily hold and therefore every maximal sequentially compact space is not an $\mathscr{L}^{*}$-space. 
EXAMPLE 6.1. An $E_{0}$ maximal sequentially compact space which is not $E_{1}$ and is not an $\mathscr{L}^{*}$-space.

Let $X=\{a, b\} \cup\{(n, 0): n \in N\} \cup\{(n, m): n, m \in N\}$. The topology $T$ on $X$ is generated by the following neighborhood bases:

$U((n, m))=\{(n, m)\}, n, m \in N$;

$U_{r}((n, 0))=\{(n, 0)\} \cup\{(n, m): m \geqq r\}, r \in N$;

$U_{r}(a)=\{a\} \cup\{(n, m): n \geqq r, m \in N\}, r \in N$;

$U_{r}(b)=\{b\} \cup\left\{(n, m): n \geqq r, m \geqq a_{n}\right\} \cup\{(n, 0): n \geqq r\}$ where $a_{n}$ varies with each $r$ and for each $n$ in the particular neighborhood, $r \in N$.

It is easily verified that the space is maximal sequentially compact and is not an $\mathscr{L}^{*}$-space since $\mathrm{cl}(N \times N)=X$ and no sequence in $N \times N$ converges to $b$.

C. E. Aull [4] showed that maximal sequentially compact spaces are such that every sequentially closed set is closed. A set is sequentially closed if it contains the sequential limits of all convergent sequences. Thus even if convergent sequences have unique limits and the sequentially closed sets are closed (C. E. Aull's condition $\mathrm{S}_{5}$ ), a space is not necessarily an $\mathscr{L}^{*}$-space.

\section{Maximal Lindelöf.}

EXAMPLE 7.1. Countable discrete spaces are maximal Lindelöf.

EXAMPLE 7.2. This is an example in [11]. Let $X$ be an uncountable set and choose a point $x_{0} \in X$. The topology $T$ on $X$ is generated by having all sets $\{x\}$ open for $x \neq x_{0}$, and if $x_{0} \in G$ then $G$ is open if $X-G$ is countable. $(X, T)$ is maximal Lindelöf.

We have as a consequence of Theorem 2.4 that a Lindelöf space is maximal Lindelöf if and only if the Lindelöf subsets are precisely the closed sets and Corollary 2.1 states that maximal Lindelöf spaces are $T_{1}$.

A topological space $(X, T)$ is a quasi-P-space if the space is $T_{1}$ and every $G_{\delta}$ is open. A topological space $(X, T)$ is a $P$-space if it is quasi- $P$ and completely regular.

The following results follow immediately from the definitions, and L. Gillman and M. Jerison [11] stated these results for $P$-spaces.

THEOREM 7.1. In a quasi-P-space every countable subset is closed and discrete in its relative topology.

THEOREM 7.2. In a quasi-P-space every subspace is quasi-P.

THEOREM 7.3. Every countable quasi-P-space is discrete.

The following result was proved by J. C. Smith, Jr.

THEOREM 7.4. Every maximal Lindelöf space is a quasi-P-space.

Proof. The theorem follows from the fact that the countable union of Lindelöf subspaces is Lindelöf. 
The converse of the preceding theorem is false since an uncountable discrete space is a $P$-space but is not Lindelöf.

THEOREM 7.5. Every Hausdorff maximal Lindelöf space is a normal P-space.

Proof. Let $A$ be open and $x \in A$. Then $X-A$ is Lindelöf, and for $y \in X-A$ there exist open neighborhoods $U_{y}(x)$ and $U_{x}(y)$ of $x$ and $y$ respectively such that $U_{y}(x) \cap U_{x}(y)=\varnothing$. Then $\left\{U_{x}(y): y \in X-A\right\}$ is an open cover of $X-A$ and there exist $y_{i}, i \in N$, such that $\left\{U_{x}\left(y_{i}\right): i \in N\right\}$ is an open subcover. $U=\bigcap\left\{U_{y_{i}}(x): i \in N\right\}$ is open and $\mathrm{cl}_{T} U \cap(X-A)=\varnothing$. Therefore the space is regular and since it is Lindelöf and regular it is normal and thus completely regular.

\section{COROLlaRY 7.1. In a Hausdorff quasi-P-space, Lindelöf subsets are closed.}

The following example of a maximal Lindelöf space is a modification of Hing Tong's example [30], and shows that not all maximal Lindelöf spaces are Hausdorff, and therefore not all maximal Lindelöf spaces are $P$-spaces.

EXAmple 7.3. Let $X$ be the Cartesian plane together with two distinct points $a$ and $b$. The topology $T$ on $X$ is defined by the following basic neighborhood systems:

(1) $\{(r, s)\}$ is open for each point $(r, s)$ in the plane;

(2) for the neighborhood systems of $a$ and $b$, select two disjoint sets $A$ and $B$ such that $A \cup B=R$ and cardinality $A=$ cardinality $B=$ cardinality $R$ where $R$ is the set of real numbers; a neighborhood of $a$ is of the form

$$
\{a\} \cup\left\{(r, s): r \in A \text { and } s \in R-A_{r}\right\}
$$

where $A_{r}$ is an arbitrarily chosen countable subset of $R$ and differs with each $r \in A$ and each neighborhood of $a$;

(3) a neighborhood of $b$ is of the form

$$
\{b\} \cup\{(r, s): r \in R-(C \cup D)\} \cup\left\{(r, s): r \in C \text { and } s \in R-C_{r}\right\}
$$

where $C$ and $D$ are arbitrarily chosen countable subsets of $B$ and $A$ respectively; $C_{r}$ is an arbitrarily chosen countable subset of $R$ which differs with each $r \in C$; and $C, C_{r}$ and $D$ differ with each neighborhood of $b$.

THEOREM 7.6. Every Lindelöf Hausdorff quasi-P-space is maximal Lindelöf and minimal Hausdorff quasi-P.

Proof. By Corollary 7.1, the Lindelöf subsets are closed and so the space is maximal Lindelöf. If any weaker space is Hausdorff quasi- $P$ it is also Lindelöf and therefore it would be maximal Lindelöf which is a contradiction.

COROLlary 7.2. Every Lindelöf P-space is maximal Lindelöf and minimal P.

Theorem 7.6 raises the question of whether or not we can say that a space weaker than a maximal Lindelöf Hausdorff space is not Hausdorff or not quasi- $P$; that is: Is a Lindelöf $P$-space minimal Hausdorff or minimal quasi- $P$ ? The following examples show that the answer to both of these questions is in the negative. 
EXAMPLE 7.4. Let $(X, T)$ be the space of Example 7.2. Let $S$ be an infinite subset of $X$ and $x_{0} \notin S$. Then $S$ is open and closed but is not minimal Hausdorff and therefore the space is not minimal Hausdorff since M. P. Berri [5] showed that if a subspace of a minimal Hausdorff space is both open and closed then it is minimal Hausdorff.

EXAMPLE 7.5. Let $(X, T)$ be the space of Example 7.2. Let $x_{1} \neq x_{0}$ and weaken the topology $T$ by requiring that all neighborhoods of $x_{1}$ have countable complements. This space is weaker than $(X, T)$ and is quasi- $P$.

We shall investigate minimal Hausdorff quasi- $P$, minimal quasi- $P$, and minimal $P$-spaces in more detail in $\$ \S 8,9$, and 10.

We know by Theorem 2.5 that Lindelöf subsets of maximal Lindelöf spaces are maximal Lindelöf in their relative topologies. However, a maximal Lindelöf subspace of a Lindelöf space does not have to be closed. In Example 7.5 the subset $X-\left\{x_{1}\right\}$ is maximal Lindelöf but is not closed in $(X, T)$.

Hausdorff quasi- $P$ quotients of Lindelöf spaces are maximal Lindelöf. However, the identity map defined on the Lindelöf space of Example 7.5 yields a quotient space which is quasi- $P$ but not maximal Lindelöf.

We showed in Theorem 7.4 that every maximal Lindelöf space is a quasi- $P$-space; therefore not all Lindelöf spaces are strongly Lindelöf. For example, the reals with the usual topology cannot have a strictly stronger maximal Lindelöf topology since every point is a $G_{\delta}$ and the reals are uncountable. This fact is also observed as a result of the following theorem.

THEOREM 7.7. If $(X, T)$ is a Lindelöf space which has a cover of $G_{\delta}$ sets which has no countable subcover, then $(X, T)$ is not strongly Lindelöf.

COROLlaRY 7.3. If $(X, T)$ is a Lindelöf $E_{0}$ space, then $(X, T)$ is strongly Lindelöf if and only if $X$ is countable.

It is well known that products of Lindelöf spaces are not necessarily Lindelöf; the best known example is the real numbers with the half open interval topology and we note that this space-being stronger than the real numbers with the usual topology-does not have a strictly stronger maximal Lindelöf topology. By Corollary 7.3, the Cantor set is not strongly Lindelöf; so, by Theorem 2.12, the infinite product of $T_{1}$ spaces of more than one point is never strongly Lindelöf. The proof that the finite product of maximal Lindelöf Hausdorff spaces is maximal Lindelöf Hausdorff is delayed to $\$ 10$.

The following theorem describes sequences in quasi- $P$-spaces and shows that sequences are of little use in these spaces and therefore in maximal Lindelöf spaces.

THEOREM 7.8. In a quasi-P-space the following hold:

(a) A sequence $\left(s_{n}\right)$ converges to $b$ if and only if there is a $k \in N$ such that for all $n \geqq k, s_{n}=b$.

(b) A sequence $\left(s_{n}\right)$ has an adherent point $b$ if and only if there is a subsequence $\left(s_{n_{k}}\right)$ such that $s_{n_{k}}=b$ for all $n_{k}$. 
Both results follow immediately from the fact that in a quasi- $P$-space, for any element $b$ which does not appear an infinite number of times in the sequence, there is a neighborhood of $b$ which contains only finitely many terms of the sequence.

Some properties closely associated with Lindelöf spaces are those of separability and first and second countability. The following theorems show that these properties are of little importance in maximal Lindelöf spaces.

THEOREM 7.9. A maximal Lindelöf space is first countable, second countable, or separable if and only if it is countable.

Proof. The proof follows immediately from Corollary 7.3 or Theorem 7.1.

THEOREM 7.10. A maximal Lindelöf space $(X, T)$ is compact, countably compact, or sequentially compact if and only if $X$ is finite.

Proof. If $X$ is finite, then the space has the three properties. If $X$ is infinite, there is a closed countably infinite subset which is discrete in its relative topology and so is not compact, countably compact, or sequentially compact.

THEOREM 7.11. Let $(X, T)$ be a quasi-P-space such that $X=\bigcup\left\{B_{i}: i \in N\right\}$ and $\left(B_{i}, T / B_{i}\right)$ is Lindelöf for $i \in N$. Then $(X, T)$ is maximal Lindelöf if and only if $B_{i}$ is $T$ closed and $\left(B_{i}, T / B_{i}\right)$ is maximal Lindelöf for each $i \in N$.

L. Gillman and M. Jerison [11] have shown that $P$-spaces have bases of open and closed sets and are therefore totally disconnected, but maximal Lindelöf spaces are not necessarily totally disconnected. A totally disconnected maximal Lindelöf space is Hausdorff.

Let $(X, T)$ be a non-Lindelöf space and $x^{*} \notin X$. Let $X^{*}=\left\{x^{*}\right\} \cup X$ and $T^{*}$ the topology on $X^{*}$ such that if $G \in T$ then $G \in T^{*}$ and if $x^{*} \in G \in T^{*}$ then $X^{*}-G$ is a closed Lindelöf subset of $(X, T)$. The space $\left(X^{*}, T^{*}\right)$ is called the one point Lindelöf extension of $(X, T)$. We are interested in the conditions for the one point Lindelof extension to be maximal Lindelöf. We know that the space $(X, T)$ must be a quasi- $P$ space since subspaces of quasi- $P$-spaces are quasi- $P$-spaces. To aid in our investigation, we define the concept of locally Lindelöf.

There are three definitions for locally Lindelöf in keeping with the literature:

(i) Every point has a Lindelöf neighborhood.

(ii) Every point has a neighborhood whose closure is Lindelöf.

(iii) Every neighborhood of a point contains a neighborhood whose closure is Lindelöf.

A space which satisfies condition (iii) satisfies condition (ii), and a space which satisfies condition (ii) satisfies condition (i). A Hausdorff quasi- $P$-space satisfying condition (i) satisfies (iii).

THEOREM 7.12. The one point Lindelöf extension of a locally Lindelöf Hausdorff quasi-P-space is a Lindelöf $P$-space and therefore is maximal Lindelöf. 
Proof. It follows immediately that the space is Hausdorff and Lindelöf. Let $x^{*}$ denote the added point; we need to show that the space is quasi- $P$ and the theorem follows; that is, we just need to show that any $G_{\delta}$ containing $x^{*}$ is open. Let $x^{*} \in \bigcap\left\{G_{i}: i \in N\right\}, G_{i} \in T^{*}$ for $i \in N$. Then $X-G_{i}$ is a closed Lindelöf subset of $X$ for each $i \in N$ and $\bigcup\left\{X-G_{i}: i \in N\right\}$ is Lindelöf and therefore closed. Thus $\bigcap\left\{G_{i}: i \in N\right\}$ is $T^{*}$ open and so the space is quasi- $P$.

COROLlary 7.4. A locally Lindelöf Hausdorff quasi-P-space is a P-space.

The following example is a maximal Lindelöf Hausdorff space with $B$ nonisolated points, $B$ an arbitrary cardinal number. This example was determined independently by J. R. Porter.

EXAMPLE 7.6. Let $(X, T)$ be the free union of $B$ copies of the maximal Lindelöf space of Example 7.2. Then $(X, T)$ is locally Lindelöf; and since it is a Hausdorff quasi- $P$-space, it is maximal Lindelöf if $B$ is countable. If $B$ is uncountable, then the one point Lindelöf extension of $(X, T)$ is maximal Lindelöf. For $B=$ cardinality $X$ the cardinality of the nonisolated points is the same as the cardinality of the isolated points, and the isolated points are dense in $X$.

The hypothesis of the following theorem is necessary since not every free $z$-filter with the countable intersection property is contained in a $z$-ultrafilter with the countable intersection property [11].

THEOREM 7.13. Let $(X, T)$ be a completely regular Hausdorff space such that every free $z$-filter with the countable intersection property is contained in a $z$-ultrafilter with the countable intersection property, then the real compactification $\nu X$ is Lindelöf.

Proof. Let $Z=\left\{z_{\alpha}: \alpha \in A\right\}$ be a $\nu X$-filter with the countable intersection property. Then $Z^{*} \doteq\left\{z_{\alpha} \cap X: \alpha \in A\right\}$ is an $X z$-filter with the countable intersection property. If $Z^{*}$ is fixed then $Z$ is fixed. If $Z^{*}$ is not fixed, then it is contained in a $z$-ultrafilter $F$ with countable intersection property. There is $x \in \nu X-X$ such that $F$ converges to $x$ which implies that $x \in z_{\alpha}$ for all $\alpha \in A$ and therefore $Z$ is fixed. Thus in $\nu X$ every $z$-filter with the countable intersection property is fixed which is equivalent to saying that $\nu X$ is Lindelöf.

EXAMPLE 7.7. Assuming the continuum hypothesis let $X$ be an $n_{1}$ set of cardinality $c$ and let $T$ be the order topology. Then $(X, T)$ is a $P$-space [11] but is not locally Lindelöf. Using the fact that each point $x$ is the limit point of a cofinal, wellordered subset of type $w_{1}$ [11, Exercise 13I(1)] and that a convergent, cofinal, well-ordered subset of type $w_{1}$ plus its limit point in an $n_{1}$ space is closed and is Lindelöf, it can be seen that the one point Lindelöf extension of $(X, T)$ is not Hausdorff but is maximal Lindelöf.

COROllary 7.5. If $(X, T)$ is a P-space such that every $z$-filter with the countable intersection property is contained in a z-ultrafilter with the countable intersection property, then $\nu X$ is maximal Lindelöf. 
Proof. $\nu X$ is a $P$-space since $X$ is a $P$-space (Exercise 8A in [11]) and is Lindelöf by the preceding theorem.

\section{Minimal quasi- $P$-spaces.}

THEOREM 8.1. The minimal quasi-P-spaces are the spaces in which the only nonempty open sets are those sets with countable complements.

Proof. Follows from Theorem 7.1.

R. E. Larson [16] has characterized minimum spaces and his characterization shows that the minimal quasi- $P$-spaces are minimum quasi- $P$-spaces.

IX. Minimal Hausdorff quasi- $P$-spaces. The proofs of all theorems in this section are either straightforward or similar to proofs in the Hausdorff case [5], [7], [13], [20], [21], [25], [27], [34].

A filter (base) is real if every countable intersection of members of the filter (base) is in the filter (generated by the filter base); that is, the filter (generated by the filter base) is closed under countable intersections.

A Hausdorff quasi- $P$-space is $L$-closed if it is closed in every Hausdorff quasi- $P$ space in which it may be embedded.

A topological space $(X, T)$ has property

$\left(\mathrm{L}_{1}\right)$ if every open cover of $(X, T)$ has a countable subfamily whose closures are a cover;

$\left(\mathrm{L}_{2}\right)$ if every open cover of $(X, T)$ has a countable subfamily whose union is dense [10];

$\left(\mathrm{L}_{3}\right)$ if every real open filter base on $(X, T)$ has at least one adherent point;

$\left(\mathrm{L}_{4}\right)$ if every real open filter base with unique adherent point is convergent.

These properties satisfy the following:

(a) A space with property $L_{1}$ has property $L_{2}$ and a space with property $L_{2}$ has property $\mathrm{L}_{3}$.

(b) In a quasi- $P$-space, $\mathrm{L}_{1}, \mathrm{~L}_{2}$ and $\mathrm{L}_{3}$ are equivalent.

(c) In a Hausdorff quasi- $P$-space, $\mathrm{L}_{1}, \mathrm{~L}_{2}, \mathrm{~L}_{3}$ and $L$-closed are equivalent.

(d) A regular space with property $L_{1}$ is Lindelöf.

(e) $\mathrm{L}_{3}$ is preserved under continuous mappings.

(f) A Hausdorff quasi- $P$-space with property $\mathrm{L}_{4}$ has property $\mathrm{L}_{1}$.

(g) A regular, $L$-closed space is maximal Lindelöf and a $P$-space.

THEOREM 9.1. If products of topological spaces are $\mathrm{L}_{3}$ then each component space is $\mathrm{L}_{3}$.

Let $(X, T)$ be a topological space and $\beta$ a base for $T$. Then $T(\beta)$ is the topology on $X$ generated by all sets of the form $X-\mathrm{cl}_{T} H$ where $H \in \beta ; T(\beta)$ is called the co-topology of $T$ with respect to the base $\beta$. 
THEOREM 9.2. The following conditions are equivalent in a Hausdorff quasi-Pspace $(X, T)$ :

(1) $(X, T)$ is minimal Hausdorff quasi-P;

(2) $(X, T)$ is $\mathrm{L}_{4}$;

(3) $\varnothing=\{T(\beta): T(\beta) \subset T, T(\beta)$ is Hausdorff quasi- $P$, and $\beta$ is a base for $T\}$;

(4) $(X, T)$ is semiregular and $\mathrm{L}_{3}$.

THEOREM 9.3. Finite products of Hausdorff quasi-P-spaces are minimal Hausdorff quasi-P if and only if each coordinate space is minimal Hausdorff quasi-P.

COROllaRY 9.1. Finite products of Katetov Hausdorff quasi-P-spaces are Katetov Hausdorff quasi-P.

Minimal Hausdorff quasi- $P$-spaces are not necessarily extremely disconnected since the maximal Lindelöf space of Example 7.2 is not extremely disconnected.

EXAMPLE 9.1. This is an example of a minimal Hausdorff quasi- $P$-space which is not regular and is therefore not Lindelöf and is not a $P$-space. This is a modification of an example of P. Urysohn [32].

Let $X=\{c, d\} \cup[(0,1) \times(-1,-1)]$. The topology $T$ on $X$ is defined by the following basic neighborhoods:

$U((m, n))=\{(m, n)\}$ for $n \neq 0$;

$U((m, 0))=\{(m, 0)\} \cup\{(m, n): n \in(-1,1)-A\}$ where $A$ is an arbitrary countable subset of $(-1,1)$ which varies with each neighborhood of $(m, 0)$;

$U(c)=\{c\} \cup\{(m, n): m \in(0,1)-B, n \in(0,1)\}$ where $B$ is an arbitrary countable subset of $(0,1)$ and varies with each neighborhood of $c$; and

$U(d)=\{d\} \cup\{(m, n): m \in(0,1)-C, n \in(-1,0)\}$ where $C$ is an arbitrary countable subset of $(0,1)$ and varies with each neighborhood of $d$.

EXAMPLE 9.2. Let $(X, T)$ be the space of the preceding example and let $(Y, U)$ be the subspace $\{d\} \cup(0,1) \times(-1,0]$ with the relative topology. The mapping $f:(X, T) \rightarrow(Y, U)$ defined below is open and continuous:

$f(c)=d ; f(d)=d ; f((m, 0))=(m, 0)$;

$f((m, n))=(m, n)$ for $n<0$; and

$f((m, n))=(m,-n)$ for $n>0$.

$(Y, U)$ is Hausdorff quasi- $P$ but is not minimal Hausdorff quasi- $P$ since it is not $L_{4}$. The real open filter base $F^{*}=\{F: F=[(0,1)-A] \times(-1,0], A$ an arbitrary countable subset of $(0,1)\}$ has a unique adherent point $d$, but it does not converge to $d$.

The preceding example shows that $L_{3}$ spaces are not necessarily $L_{1}$ and so $L_{3}$ spaces are not necessarily minimal Hausdorff quasi- $P$-spaces. The example also shows that $\mathrm{L}_{4}$ is not preserved under continuous open surjections.

EXAMPLE 9.3. This is an example of a closed subset of minimal Hausdorff quasi- $P$-space which is not minimal Hausdorff quasi- $P$ and is not $L_{3}$.

Let $A=\{(m, 0): m \in(0,1)\}$ with the relative topology of Example 9.1 ; that is, 
the discrete topology. $A$ is closed in the space but is not closed in its one point Lindelöf extension and so is not $L$-closed.

\section{Minimal $P$-spaces.}

Lemma 10.1. Let $(X, T)$ be a $P$-space and $F^{*}$ a real $z$-filter without adherent points. Then the space $\left(X, T^{*}\right)$ with $T^{*}=\left\{U \in T: x_{0} \in U\right.$ implies $F \subseteq U$ for some $\left.F \in F^{*}\right\}\left(x_{0}\right.$ an arbitrarily chosen point of $\left.Z\right)$ is a $P$-space.

TheOREM 10.1. A P-space $(X, T)$ is minimal $P$ if and only if it is Lindelöf.

Proof. We have previously shown (Corollary 7.2 ) that every Lindelöf $P$-space is minimal $P$. If $(X, T)$ is not a Lindelöf $P$-space, then there is a real $z$-filter without adherent points and by the preceding lemma the space is not minimal $P$.

Every minimal $P$-space is minimal Hausdorff quasi- $P$ since every Lindelöf Hausdorff quasi- $P$-space is a $P$-space but every minimal Hausdorff quasi- $P$-space is not minimal $P$ as shown by Example 9.1.

THEOREM 10.2. Finite products of maximal Lindelöf Hausdorff spaces (minimal $P$-spaces) are maximal Lindelöf (minimal $P$ ).

Proof. We have shown that finite products of minimal Hausdorff quasi- $P$-spaces are minimal Hausdorff quasi- $P$, and since products of regular spaces are regular and regular minimal Hausdorff quasi- $P$-spaces are Lindelöf, the result follows.

COROLlaRY 10.1. Let $\left(X_{\alpha}, T_{\alpha}\right)$ be Lindelöf spaces for which there exist finer maximal Lindelöf Hausdorff topologies, $\alpha \in A, A$ finite. Then $\left(\prod_{A} X_{\alpha}, \prod_{A} T_{\alpha}\right)$ is strongly Lindelöf.

Theorem 10.3. A P-space is closed in any P-space in which it may be embedded if and only if it is L-closed.

The proof follows from the fact that a $P$-space is closed in any $P$-space in which it may be embedded if and only if every real $z$-filter has nonvoid adherence.

TheOREM 10.4. A P-space $(X, T)$ can be embedded as a dense open subset of a Lindelöf $P$-space if and only if it is locally Lindelöf.

Proof. If the space is locally Lindelöf, its one point Lindelöf extension is a Lindelöf $P$-space. If it can be densely embedded as an open subset of a Lindelöf $P$-space, then for $x \in X$ there is an open and closed subset of $Y$ containing $x$ and contained entirely within $X$ and it is Lindelöf.

Theorem 10.5. Let $(X, T)$ be a $P$-space. Then $X$ is minimal $P$ only if every $X$ free real $z$-filter is contained in a real $z$-ultrafilter.

The proof follows from Theorems 7.13 and 10.1 .

\section{BIBLIOGRAPHY}

1. P. Alexandrov and P. Urysohn, Une condition nécessaire et suffisante pour qu'une classe $(\mathscr{L})$ soit une classe $(\mathscr{D})$, C. R. Acad. Sci. Paris 177 (1923), 1274-1276.

2. C. E. Aull, A certain class of topological spaces, Prace Mat. 11 (1967), 49-53. MR 37 \#3498. 
3. C. E. Aull, Sequences in topological spaces, Notices Amer. Math. Soc. 12 (1965), 222. Abstract \#65T-79.

4. - Sequences in topological spaces, Prace Mat. 11 (1968), 329-336. MR 37 \#2169.

5. M. P. Berri, Minimal topological spaces, Trans. Amer. Math. Soc. 108 (1963), 97-105. MR 27 \#711.

6. M. P. Berri, J. R. Porter and R. M. Stephenson, Jr., General topology and its relation to modern analysis and algebra. III, Proc. Conference Topology (Kanpur, 1968), Academic Press, New York, 1970.

7. N. Bourbaki, Espaces minimaux et espaces completement séparés, C. R. Acad. Sci. Paris 212 (1941), 215-218.

8. — Topologie générale, 3rd ed., Actualités Sci. Indust., no. 1142, Hermann, Paris, 1961. MR 25 \#4480.

9. M. Fréchet, Sur quelques points du calcul fonctionnel, Rend. Circ. Mat. Palermo 22 (1906), 1-74.

10. Z. Frolik, Generalisations of compact and Lindelöf spaces, Czechoslovak Math. J. 9 (84) (1959), 172-217. (Russian) MR 21 \#3821.

11. L. Gillman and M. Jerison, Rings of continuous functions, University Series in Higher Math., Van Nostrand, Princeton, N. J., 1960. MR 22 \#6994.

12. E. Hewitt, A problem of set theoretic topology, Duke Math. J. 10 (1943), 309-333. MR 5, 46.

13. Shogo Ikenaga, Product of minimal topological spaces, Proc. Japan Acad. 40 (1964), 329-331. MR 29 \#5213.

14. J. E. Joseph, Continuous functions and spaces in which compact sets are closed, Amer. Math. Monthly 76 (1969), 1125-1126. MR 40 \#8018.

15. C. Kuratowski, Topologie. Vol. I, 3rd ed., PWN, Warsaw, 1952; English transl., Academic Press, New York; PWN, Warsaw, 1966. MR 14, 1000.

16. R. E. Larson, Minimum and maximum topological spaces, Notices Amer. Math. Soc. 16 (1969), 847. Abstract \#69T-G99.

17. N. Levine, Simple extensions of topologies, Amer. Math. Monthly 71 (1964), 22-25. MR 29 \#580.

18. - When are compact and closed equivalent?, Amer. Math. Monthly 72 (1965), 41-44.

19. A. S. Parhomenko, Über eineindeutige stetige Abbildungen, Mat. Sb. 5 (47) (1939), 197210. (Russian) MR 1, 221.

20. J. R. Porter, Minimal first countable spaces, Bull. Austral. Math. Soc. 3 (1970), 55-64.

21. J. R. Porter and J. Thomas, On H-closed and minimal Hausdorff spaces, Trans. Amer. Math. Soc. 138 (1969), 159-170. MR 38 \#6544.

22. A. Ramanathan, A characterization of maximal-Hausdorff spaces, J. Indian Math. Soc. 11 (1947), 73-80. MR 10, 137.

23. — , Maximal-Hausdorff spaces, Proc. Indian Acad. Sci. Sec. A 26 (1947), 31-42. MR 9, 98.

24. — - Minimal-bicompact spaces, J. Indian Math. Soc. 12 (1948), 40-46. MR 10, 390; 856.

25. C. T. Scarborough and A. H. Stone, Products of nearly compact spaces, Trans. Amer. Math. Soc. 124 (1966), 131-147. MR 34 \#3528.

26. N. Smythe and C. A. Wilkins, Minimal Hausdorff and maximal compact spaces, J. Austral. Math. Soc. 3 (1963), 167-171. MR 27 \#4204.

27. R. M. Stephenson, Jr., Minimal first countable topologies, Trans. Amer. Math. Soc. 138 (1969), 115-127. MR 38 \#6537. 
28. J. Pelham Thomas, Maximal connected topologies, J. Austral. Math. Soc. 8 (1968), 700-705. MR 38 \#5177.

29. W. J. Thron, Topological structures, Holt, Rinehart and Winston, New York, 1966. MR 34 \#778.

30. Hing Tong, Note on minimal bicompact spaces (preliminary report), Bull. Amer. Math. Soc. 54 (1948), 478-479.

31. P. Urysohn, Sur les classes $(\mathscr{L})$ de M. Fréchet, Enseignement Math. 25 (1926), 77-83.

32. — U Uber die Machtigkeit der zusammenhangen Mengen, Math. Ann. 94 (1925), 262-295.

33. R. Vaidyanathaswamy, Set topology, Chelsea, New York, 1947.

34. G. Vigilino, A co-topological application to minimal spaces, Pacific J. Math. 27 (1969), 197-200.

The University OF AKron,

AKron, OHIO 44304 